\title{
Study on Dynamic Characteristics and Improvement of the Current Collector in High-speed Metro Vehicles
}

\author{
Hongfei Meng1, a, Xiukun Wei ${ }^{2, b, ~}{ }^{*}$, Yongjun $\mathrm{Liu}^{3, \mathrm{c}}$ \\ ${ }^{1}$ State Key Laboratory of Rail Traffic Control and Safety, Beijing Jiao tong University, Beijing 100044, \\ China; \\ ${ }^{2}$ School of Traffic and Transportation, Beijing Jiao tong University, Beijing 100044, China; \\ ${ }^{3}$ School of Mechanical, Electronic and Control engineering, Beijing Jiao tong University, Beijing \\ 100044, China. \\ a17120966@bjtu.edu.cn, b, *xkwei@bjtu.edu.cn, cyjliu@bjtu.edu.cn
}

Keywords: Current collector; third rail; dynamic response; Adams simulation.

\begin{abstract}
With the increasing of the maximum running speed and the complicated environment that the current collector is exposed to, it is urgent and necessary to study the dynamic characteristics of the current collector under the high-speed running condition. In this paper, the current collector used in the vehicle of Guangzhou Metro Line 14 (the maximum running speed is $120 \mathrm{~km} / \mathrm{h}$ ) is considered as the object of the study. The irregularity of the third rail is used as the input excitation and the random vibration is considered while the vehicle is running. Four factors which include the stiffness and the damping of the tension spring, the length of the swing arm, as well as the gravity center position of the shoe are analyzed on their impact to the dynamic response of the current collector at the speed of $120 \mathrm{~km} / \mathrm{h}$. The contact force of the current collector and the third rail, the displacement and the vertical acceleration of the shoe are considered in the dynamic characteristics. As a consequence, the influence of different factors on the quality of current collecting is analyzed and the improvement of the optimal design is put forward. The simulation results demonstrate that the improved collector can perform better in the quality of current collecting than before.
\end{abstract}

\section{Introduction}

Nowadays, the urban rail transit especially the metro has been paid more and more attention in our daily travel. Not only a good current-collecting condition is a prerequisite for ensuring the safe, reliable and stable running of the vehicle, but also it can reduce the maintenance cost of the vehicle and reduce the damage rate of the current-collector components. With the expansion of the metro line network and the increase of passenger traffic, the running speed of vehicles has also been constantly increasing, which results in higher requirements for the current-collecting performance of vehicles in high-speed and complex environments. Therefore, it is necessary to study the performance of the current collector under high speed and make some improvements in the collector.

There are two main modes for the metro vehicles to receive current, one is the pantograph-catenary system and another is the collector shoe-third rail system. Compared with the ways of receiving current by pantograph and catenary, the collector shoe-third rail system has lots of advantages, such as the lower cost, the less maintenance work, the longer service life and less impact on the city landscape on the ground. Therefore, more and more newly-built subway lines begin to adopt the third rail system, which attracts lots of researches in the world. The contact style between the collector shoe and the third rail includes three modes, they are upper contact, side contact and lower contact. In this paper, the lower contact style collector with a tension spring is studied.

In the last decade, the current collector shoe as well as the third rail has attracted the attention of researchers all over the world (see [1]-[6]). In [1], the data of the contact force and the displacement of the collector shoe are analyzed and the vibration rule of the shoe is obtained by establishing the circular third rail test rig combined with the vehicle test device. In [2], some sensors are installed between the bogie and the current collector. The result indicates that the hysteresis of the collector 
shoe can cause the shoe off-line from the third rail. By establishing the matching characteristic motion model of the contact surface between the collector and the third rail [3], the motion characteristics of the slider are analyzed and the factors that affect the running speed of the collector are identified. In [4], taking the lower contact collector in the vehicle of Shanghai Metro Line 11 as the research object, the vertical vibration characteristics are analyzed when the current collector pass the end elbow on the third rail. By simulating, researches [5] [6] have illustrated the characteristics of normal contact stiffness on the contact surface and analyzed the impact on the vibration response of the collector system. Up to now, most of the researches are based on the low speed (about $80 \mathrm{~km} / \mathrm{h}$ ) level, nearly no research concentrates on the high speed $(120 \mathrm{~km} / \mathrm{h})$ condition and the improvement in the study of current collector.

This paper is organized as follows. The necessity and the main aim of studying the dynamic characteristics of the collector under high speed condition are discussed in section 1 . In section 2 , the dynamic model of the collector-third rail coupling system is established and analyzed. In section 3 , by simulating in the multi-rigid body dynamics simulation software ADAMS, the dynamic responses of the collector shoe are presented and some suggestions for improvement are proposed. Finally, some conclusions are summarized in section 4.

\section{Model Building of the Collector-Third Rail Coupling System}

This section focuses on the model establishment to get the dynamic responses from the simulation. Firstly, the mathematical description of the collector-third rail system is presented. Then, a three-dimensional model of the current collector is built for the preparation of importing into the ADAMS to simulate. Finally, a preliminary simulation result is shown, which illustrates the validity of the model and the feasibility of continuing the simulations.

The simulation object is a lower contact collector, the shoe of which contacts the third rail on the bottom with the tensile force of the tension spring. In the following, vertical vibration of the shoe and the swing arm is analyzed without taking the flexible deformation produced by its installation and pressure into consideration. The schematic diagram of the collector is depicted in Fig. 1.

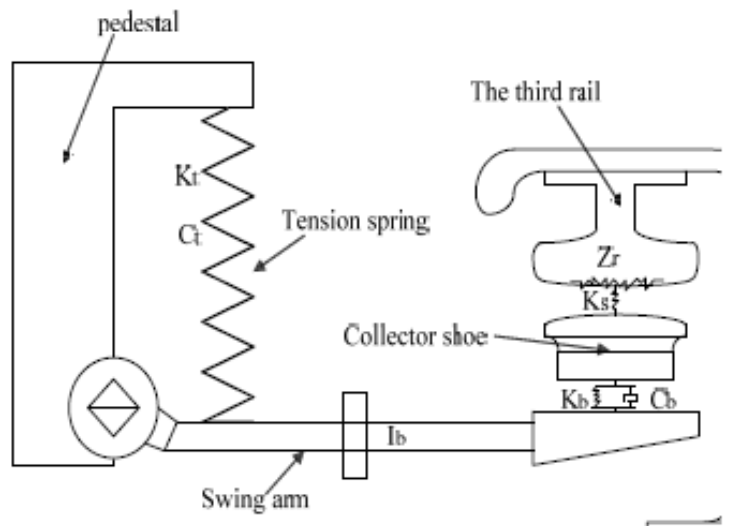

Figure. 1. The collector and the third rail coupling system

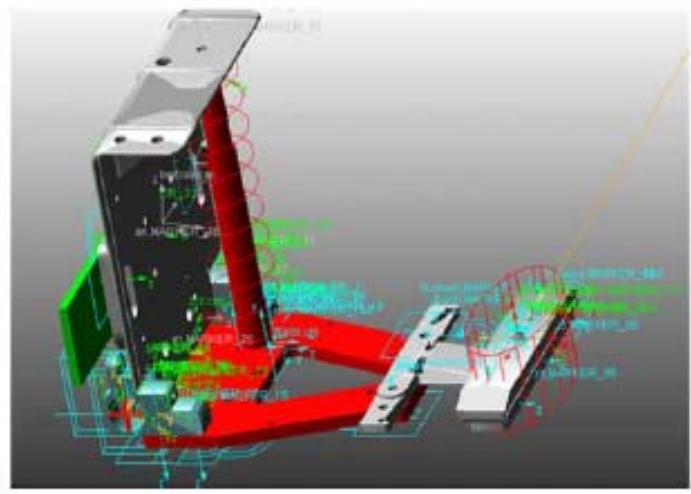

Figure.2. The collector-third rail dynamic model 
For the swing arm, the differential equation of rotational and vertical vibration can be described as:

$$
\begin{gathered}
I_{b} \ddot{\theta}_{b}=F_{b} l_{b}+m_{b} g l_{b^{\prime}}-F_{d} l_{d}-F_{p} l_{p} \\
m_{b} \ddot{Z}_{b}=F_{b}+F_{d}+m_{b} g-F_{p}
\end{gathered}
$$

Where $F_{b}, F_{d}$ and $F_{p}$ are the force between swing arm and shoe, the force between swing arm and collector base and the force of the tension spring; $l_{b}, l_{d}, l_{p}$ and $l^{\prime}{ }_{b}$ are the distance from the action point of $F_{b}, F_{d}, F_{p}$ and $m_{b} g$ to the rotation center of swing arm; $I_{b}$ and $m_{b}$ are the moment of inertia and the mass of the swing arm; $\theta_{b}$ and $Z_{b}$ are the rotation angle and the vertical vibration displacement of the arm.

For the collector shoe, the vertical vibration can be described by the formula:

$$
m_{s} \ddot{Z}_{s}=F_{s}+m_{s} g-F_{b}
$$

Where $m_{s}$ the mass of the collector is shoe; $Z_{s}$ is the vibration displacement of the shoe; $F_{s}$ is the force between the shoe and third rail.

The force of $F_{b}, F_{p}, F_{s}$ can be derived as:

$$
\begin{gathered}
F_{b}=K_{b}\left(Z_{b}-l_{b} \theta_{b}-Z_{s}\right)+C_{b}\left(\dot{Z}_{b}-l_{b} \dot{\theta}_{b}-\dot{Z}_{s}\right) \\
F_{p}=K_{t} l^{\prime}{ }_{p}+C_{t} l^{\prime}{ }_{p} \\
F_{s}=-K_{s} Z_{r}
\end{gathered}
$$

Where $K_{b}$ and $C_{b}$ denote the stiffness and damping between swing arm and shoe; $K_{t}$ and $C_{t}$ denote the stiffness and damping of the tension spring; $l^{\prime}{ }_{p}$ is the elongation of the spring; $K_{s}$ and $Z_{r}$ are the contacting stiffness and the irregularity coefficient of the third rail.

In the following, the three-dimensional model is established firstly by using SOLIDWORKS, then the dynamic parameters are added to it and the degree of freedom is constrained in ADAMS. For the deformation of the swing arm and the third rail is very small, it has little influence on the dynamic results. Thus, in this paper, the simulation model is regarded as the rigid body. To make the model move and vibrate, two places are exerted excitation. One is the base of the collector, its excitation data are gathered from the simulation in ADAMS/Rail module, in which a vehicle-rail system model is simulated and the signal on the installation point of the collector is obtained. The one is the third rail, whose irregularity is presented by using the American fifth-level power spectral density function [7] [8] [9] as an excitation.

So far, a dynamic simulation model (see, Fig. 2) of the current collector is built in the circumstance of ADAMS. The preliminary result (see, Fig. 3) shows the average contact force between the shoe and third rail is about $130 \mathrm{~N}$, which is within the normal range and indicates the model is practical and it can continue the other simulation.

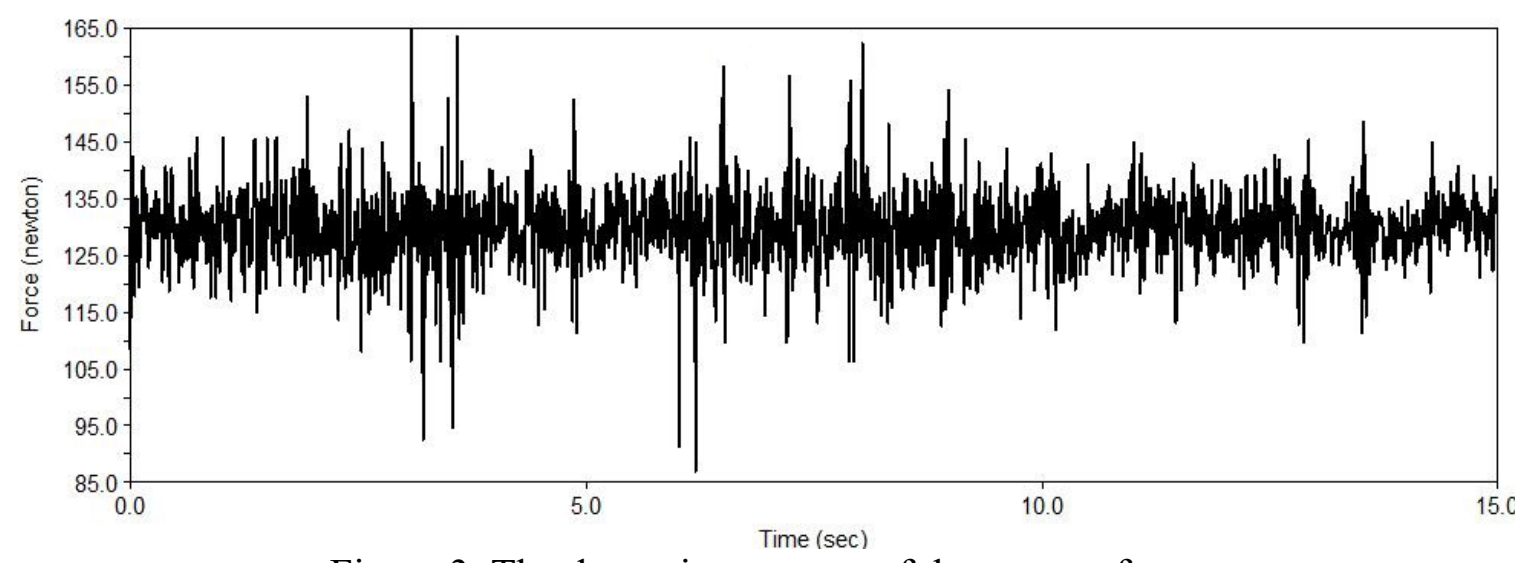

Figure.3. The dynamic response of the contact force

\section{Dynamic Responses of Tet Simulation in ADAMS}

In this section, by simulating the model in ADAMS, the dynamic simulation responses are obtained and analyzed. Four factors (the stiffness and the damping of the tension spring, the length of 
the swing arm, as well as the gravity center position of the shoe) which may influence the current collecting quality are studied to get the law of the changes. The initial parameters of the collector are listed as follows: the stiffness of the tension spring is $18200 \mathrm{~N} / \mathrm{m}$, the damping is $50 \mathrm{~N} /(\mathrm{m} / \mathrm{s})$, the length of the swing arm is $0.43 \mathrm{~m}$, and the gravity center position of the shoe in local coordinate is $5.41 \mathrm{~mm}$. After analyzing the regularity of the results, some improvements are proposed to the collector. The improved model is also established to make a simulation, the result of which indicates the improved one is much better than before in the respect of dynamic response.

For the tension spring in the collector, the stiffness changes in the formula of

$$
K^{\prime}=K_{k} \cdot K_{0}
$$

Where $K^{\prime}$ is the simulation stiffness value, $K_{0}$ is the initial stiffness of $18200 \mathrm{~N} / \mathrm{m}, K_{k}$ is the coefficient that changes from 0.1 to $0.5,1,2,5,10$.

The result is shown in Fig. 4. With the increase of the stiffness, the average value of the contact decreases and the amplitude increases gradually. The amplitude and standard deviation of the shoe displacement is decreasing within a certain range and the amplitude of acceleration is increasing slightly, which indicates the quality of current receiving is improved. But excessive increase of stiffness can make the contact force small and the standard deviation of displacement large, which will lead to higher off-line rate and affect the quality of current receiving. So the stiffness of tension spring should be increased appropriately.

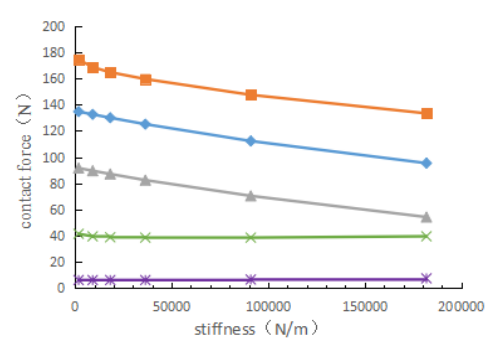

(a)

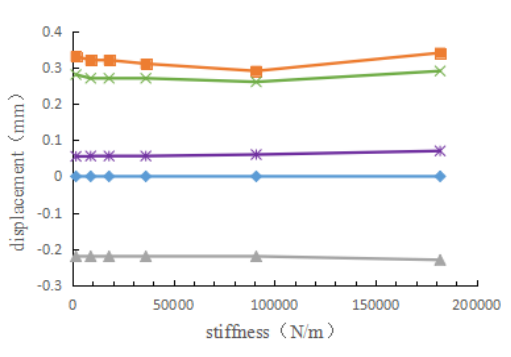

(b)

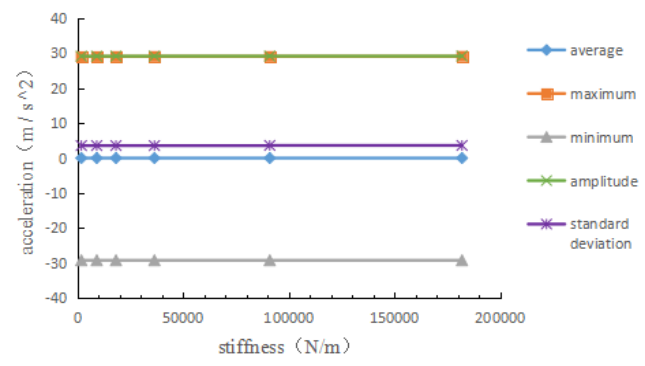

(c)

Figure 4. the dynamic responses with the change of the spring stiffness.

For the damping of the spring, it changes in the law of

$$
C^{\prime}=C_{k} \cdot C_{0}
$$

where $C^{\prime}$ is the simulation damping value, $C_{0}$ is the initial damping of $50 \mathrm{~N} /(\mathrm{m} / \mathrm{s}), C_{k}$ is the coefficient that changes from 0 to $0.1,0.5,1,2,5$.

As shown in Fig. 5, the changes of damping have little influence on the dynamic characteristics of the collector shoe. Because different from the catenary-pantograph flexible contact, the contact between collector shoe and third rail is a rigid contact and the swing arm is in a high frequency vibration. The change of spring damping $(0.1 \%-1 \%$ of the stiffness values) has little impact on the results when the stiffness is high. But excessive damping will make the contact force and the maximum displacement increase, which is not conducive to the current collecting. So it is better to reduce the damping appropriately.

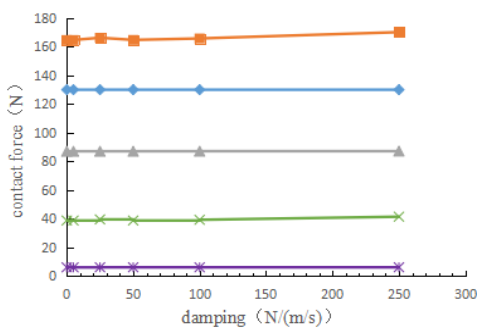

(a)

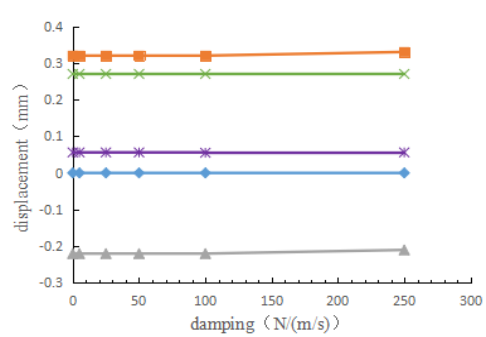

(b)

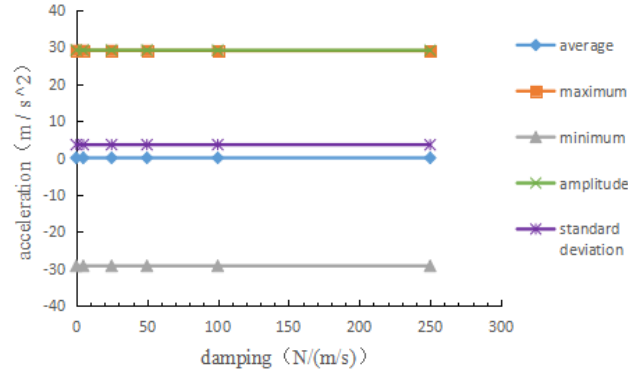

(c)

Figure 5. The dynamic responses with the change of the spring damping. 
In the simulation of changing the length of swing arm, the initial length is $0.43 \mathrm{~m}$. Then the value ranges from $0.41 \mathrm{~m}$ to $0.42 \mathrm{~m}, 0.43 \mathrm{~m}, 0.44 \mathrm{~m}, 0.45 \mathrm{~m}$. The dynamic responses (see Fig. 6 ) indicate that with the increase of the length of the swing arm, the average value of contact force will decrease and the amplitude will increase. For the acceleration, its amplitude increases and the standard deviation changes very little. Therefore, if the contact force is satisfied, increasing the length of the swing arm properly will help the current collection.

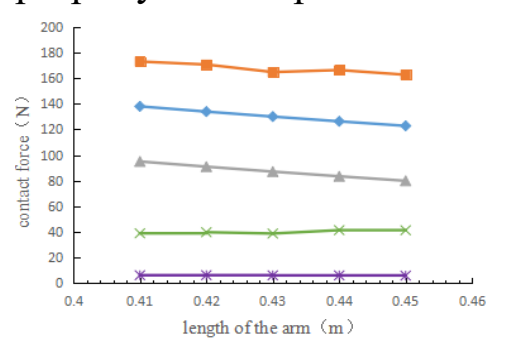

(a)

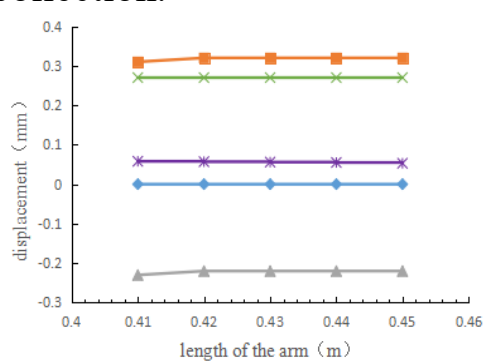

(b)

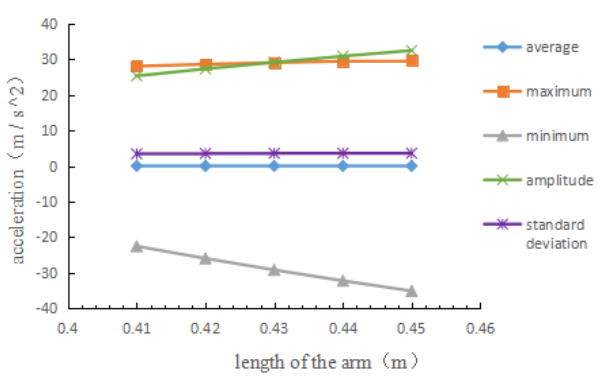

(c)

Figure 6. the dynamic responses with the change of the length of the swing arm

As for the simulation of the gravity center point of the shoe, the point in local coordinate moves from $1.41 \mathrm{~mm}$ to $3.41 \mathrm{~mm}, 5.41 \mathrm{~mm}, 7.41 \mathrm{~mm}, 10.41 \mathrm{~mm}$ while the initial value is $5.41 \mathrm{~mm}$. As depicted in the Fig. 7, when the position is higher than $5.41 \mathrm{~mm}$, the amplitude of contact force and shoe displacement become large rapidly which is unfavorable to the current collecting. The amplitude of acceleration is increased while the average value and standard deviation have little changes. Compared with the bad changes of contact force and displacement, the disadvantages are more than advantages. So it is better to maintain the original gravity center or slightly lower the position.

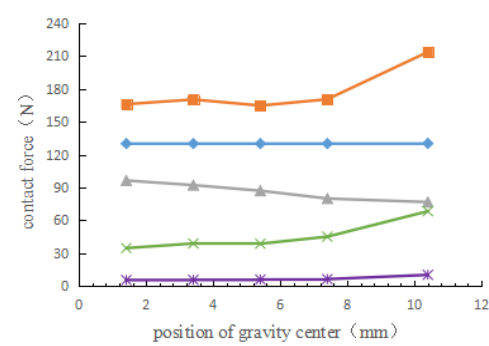

(a)

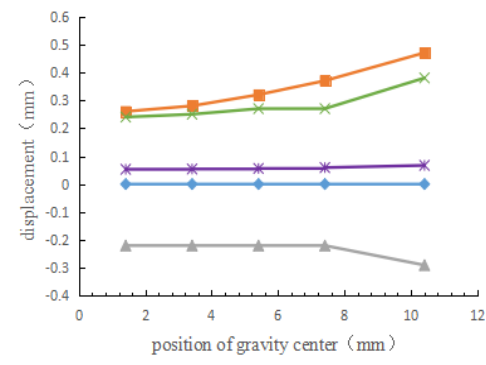

(b)

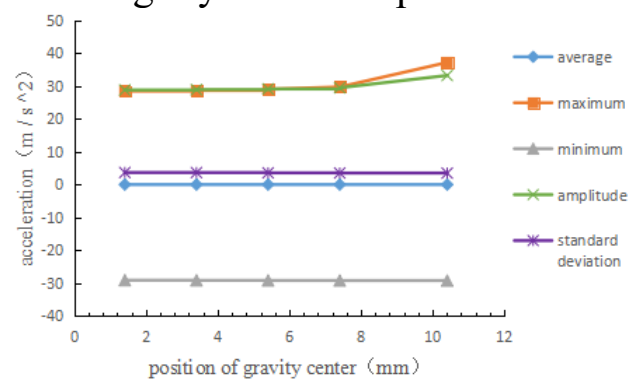

(c)

Figure 7. the dynamic responses with the change of the position of the gravity center

According to the analysis results above, the following improvements are put forward. As for the tension spring, increasing the stiffness by $30 \%$ to the value of $23660 \mathrm{~N} / \mathrm{m}$ and slightly decreasing the damping by $20 \%$ to the value of $40 \mathrm{~N} /(\mathrm{m} / \mathrm{s})$. Besides, lowering the gravity center position slightly from $5.41 \mathrm{~mm}$ to $4.41 \mathrm{~mm}$. What's more, the length of the swing arm is extended from $0.43 \mathrm{~m}$ to $0.435 \mathrm{~m}$, for there is a certain limit between the third rail and the vehicle so the length of the arm cannot be changed too much. The improved collector model was also simulated and the comparison with the previous data is shown in Table. 1.

Table. 1.The comparison of the previous and improved simulation results

\begin{tabular}{cccc}
\hline Index & $\begin{array}{c}\text { Previous } \\
\text { model }\end{array}$ & Improved model & Changes \\
\hline Average contact force $(\mathrm{N})$ & 129.91 & 126.53 & $\downarrow$ \\
Amplitude of contact force $(\mathrm{N})$ & 38.78 & 36.30 & $\downarrow$ \\
Standard deviation of contact force $(\mathrm{N})$ & 6.18 & 5.96 & $\downarrow$ \\
Amplitude of shoe displacement $(\mathrm{mm})$ & 0.27 & 0.26 & $\downarrow$ \\
Standard deviation of shoe displacement $(\mathrm{mm})$ & 0.056 & 0.054 & $\downarrow$ \\
amplitude of shoe acceleration $\left(\mathrm{m} / \mathrm{s}^{2}\right)$ & 29.07 & 29.83 & $\uparrow$ \\
Standard deviation of shoe acceleration $\left(\mathrm{m} / \mathrm{s}^{2}\right)$ & 3.56 & 3.61 & $\uparrow$ \\
\hline
\end{tabular}


From the comparison table, it is obvious that the amplitude and standard deviation of the contact force and shoe displacement are all reduced. The improved collector also meets the requirement of the dynamic contact force and ensure the low rate of being offline, which indicates the current collecting is more stable. The amplitude of acceleration increases, which illustrates that the tracking performance of the collector shoe becomes quicker and the quality of current receiving has been improved.

\section{Conclusion}

This paper has considered the current collector used in the vehicle of Guangzhou Metro Line 14 as the research object. A lower contact style collector model with a tension spring is built and simulated under high speed $(120 \mathrm{~km} / \mathrm{h})$ in the software ADAMS. Different factors have been analyzed on the impact of dynamic characteristics. In the last, the improvement measures are proposed by summing up the simulation results. Under the speed of $120 \mathrm{~km} / \mathrm{h}$, increasing the stiffness by $30 \%$, decreasing the damping by $20 \%$, lowering the gravity center position to $4.41 \mathrm{~mm}$ and extending the length of the swing arm to $0.435 \mathrm{~m}$ can make the quality of current collecting better. The conclusion has provided a reference for the research on the dynamic characteristics of high-speed subway vehicle current collectors in the future.

In this paper, only the straight track situation is analyzed and the components of the collector are regarded as the rigid bodies. To make a more accurate simulation, the curve track and rigid-flexible coupling system should be taken into consideration. This will be our future work.

\section{Acknowledgments}

This work is partly supported by Chinese National Key Project of Research and Development (Contract No. 2017YFB1201202-001).

\section{References}

[1]. P. F. Weston, E. Stewart, C. Roberts. Measuring the Dynamic Interaction between Electric Vehicle Shoe gear and the Third Rail [J]. 2008.

[2]. E. Stewart, S. Hillman Sen, C. Roberts. Using Bogie-Mounted Sensors to Understand the Dynamics of Third Rail Current Collection Systems [J]. Proceedings of the Institution of Mechanical Engineers, Part F: Journal of Rail and Rapid Transit, 2011(225/2):219-227.

[3]. Zhen Yun. Wang, Shaquan. Wang, X. Li. Collector and Contact Rail Matching Characteristic Research [J]. Modern Urban Rail Transit, 2011(06):55-57.

[4]. W. Wang. Analysis of the Contact Characteristics of the End of the Collector device and the contact rail [J]. Journal of East China Jiao tong University, 2014(01):34-38.

[5]. X. Yang. Collector and Third Rail Dynamic Contact Characteristics Research Based on Fractal Theory [D]. Beijing Jiao tong University, 2015.

[6]. G. Li. Research on Rigid-Flexible Coupling Dynamics of Third Rail Collecting System. Beijing Jiao tong University, 2015.

[7]. Y. He, Z. Li, C. Sheng. Analysis of Track Spectrum Characteristics under Different Subway Lines [J]. Journal of Railway Engineering Society, 2014(07):99-104.

[8]. F. Wang, J. Zhou, L. Ren. Track Spectrum Analysis for Dynamic Simulation of High Speed Vehicles [J]. Railway Transaction, 2002(05):21-27.

[9]. G. Qi. Research on the Vibration Response of Train Based on Track Irregularity [D]. South China University of Technology, 2014. 84. 